\title{
Heart failure with complete recovery in a patient with systemic lupus erythematosus
}

\author{
Konstantinos Lampropoulos ${ }^{1}$, Dimitrios Kotsas ${ }^{1}$, Themistoklis Iliopoulos ${ }^{2}$ \\ ${ }^{1}$ Department of Cardiology, Catheterization Laboratory, Evaggelismos General Hospital of Athens, Greece \\ 2Department of Cardiology, Catheterization Laboratory, 251 General Air Force Hospital of Athens, Greece
}

\begin{abstract}
We report the case of a 43-year-old female patient who was admitted to the Cardiology Department from the Rheumatology Clinic where she was being treated for multi-organ serositis, fatigue and mild dyspnoea on exertion. The patient had a known medical history of systemic lupus erythematosus (SLE). Following extensive evaluation with blood tests for immunological and viral culprits, cardiac ultrasound, chest and abdominal computed tomography (CT) and heart magnetic resonance imaging (MRI), the diagnosis of effusive constrictive pericarditis secondary to her SLE was made. Treatment with $\beta$-blockers, diuretics and corticosteroids was given with excellent results, and one year post discharge the patient remains asymptomatic. Systemic lupus erythematosus patients often manifest cardiac complications such as pericarditis. The practising physician should always bear in mind this possibility when treating such patients.
\end{abstract}

Key words: systemic lupus erythematosus, acute heart failure, heart failure recovery.

\section{Introduction}

Cardiac involvement is one of the main complications substantially contributing to the morbidity and mortality of patients suffering from systemic autoimmune diseases. Such involvement has been recognized since the beginning of the $20^{\text {th }}$ century, but in the last decades, newly recognized clinical entities have been described due to the introduction of very sensitive, non-invasive or semi-invasive cardiac imaging techniques [1]. All of the anatomical heart structures can be affected, and multiple pathogenic mechanisms have been reported.

Non-organ-specific autoantibodies have been implicated in immune complex formation and deposition as the initial triggers for inflammatory processes responsible for disease processes such as Libman-Sacks endocarditis, myocarditis and pericarditis. Several autoantibodies, such as anti-phospholipid antibodies (aPL), anti-SSA/Ro antibodies and anti-endothelial cell antibodies, can become potent mediators of cardiac damage. The consequences of such damage can affect several heart structures such as the valves, myocardium, pericardium, conduction tissue and cardiac arteries in patients suffering from systemic lupus erythematosus (SLE), anti-phospholipid syndrome (APS), Sjögren syndrome (SS) or other autoimmune diseases. Other cardiac manifestations, such as pericarditis, myocarditis, endocarditis and conduction disturbances, when present, are often mild and, usually, subclinical features are more prevalent than clinically apparent disease.

We report the case of a 43-year-old female with known history of SLE, who was admitted to our hospital because of mild symptoms of fatigue and dyspnoea and eventually was diagnosed with effusive constrictive pericarditis secondary to her rheumatic primary disease.

\section{Case report}

The patient was originally admitted to the Rheumatology Department because of fatigue and known medical history of SLE. Three months prior to admission, ENA, 
anti-SSA and anti-SSB antibodies were found to be elevated. ANA were also positive at that time.

She appeared haemodynamically stable with a blood pressure of $120 / 85 \mathrm{mmHg}$. The electrocardiogram (ECG) was unremarkable and heart ultrasound revealed normal left ventricular (LV) wall and cavity dimensions with mildly reduced LV systolic function. The estimated ejection fraction was 45\%, confirmed independently by two echocardiographers. The mitral valve inflow pattern was indicative of impaired relaxation ( $E$ $<A$, 'E < 'A). Right ventricle tissue Doppler imaging (TDI) indices were normal. There was also a small to medium size pericardial effusion, mainly around the right ventricle and adjacent to the anterior wall of the heart. Finally, the inferior vena cava (IVC) was dilated with no respiratory diameter variation. The patient was transferred to the Cardiology Department for in-depth follow-up of her cardiac function.

Blood tests were unremarkable apart from mild microcytic anaemia (haematocrit - Ht 33, haemoglobin $\mathrm{Hb}$ 10.6, mean corpuscular volume - MCV 76) and raised transaminase values (aspartate aminotransferase - AsPAT 175, alanine aminotransferase - AIAT 383) with marginally increased $\gamma$ glutamyl transferase $(\gamma \mathrm{GT})$ and lactate dehydrogenase $(\mathrm{LDH})$. C reactive protein (CRP) was also increased, unlike erythrocyte sedimentation rate (ESR), which was within the normal range. Cardiac troponins were modestly elevated with peak Ctnl on the day of admission 0.789 (normal < 0.056). The brain natriuretic peptide (BNP) values were also markedly elevated (up to $14700 \mathrm{pg} / \mathrm{ml}$, normal laboratory values < $100 \mathrm{pg} / \mathrm{ml}$ ). Serum total protein and albumin were modestly reduced $(3.2 \mathrm{~g} / \mathrm{dl}$, normal > 3.5). The patient was further investigated with diagnostic thoracocentesis and lab results confirmed that the aspirated pleural fluid was an exudate. A series of extensive blood tests for viral or immunologic markers had already been contacted at the Rheumatology Clinic including Coxsackie virus, cytomegalovirus (CMV), hepatitis B and C, toxoplasma and the Venereal Disease Research Laboratory (VDRL) test. Finally, a series of tests for cancer markers was also conducted without any remarkable findings apart from a marginal initial cancer antigen CA 12-5 value that was not confirmed at a second sampling a few days later. Serum amyloid A (SAA) was $10.5 \mathrm{mg} / \mathrm{l}$ (normal value $<6.4 \mathrm{mg} / \mathrm{l}$ ) and thyroid hormones were also unremarkable.

The patient was investigated with chest and abdominal CT scans. The chest scan revealed a number of lymph nodes of increased dimensions (up to $1.4 \mathrm{~cm}$ ) and a medium sized pleural effusion, already known from plain chest radiography on admission. No signs of malignancy were evident. The abdominal scan confirmed the presence of intraperitoneal fluid and also revealed a $4 \mathrm{~cm}$ cystic mass at the anatomical position of the right ovary, a finding that was considered for further evaluation.

The differential diagnosis at the time included the possibility of myocardium-involving multiple organ serositis secondary to SLE, amyloidosis, or possibly extrathoracic malignancy (e.g. Meigs syndrome) should the right ovary prove to be neoplastic tissue.

Further diagnostic work up was advised and the patient was programmed for a heart MRI and 24-hour ECG rhythm recording. The MRI showed borderline dilation of both ventricles with normal LV systolic function. No signs of acute myocardial inflammation were evident but signal enhancement in the epicardial layer of the lateral/inferolateral wall was possibly consistent with a non-ischaemic inflammatory/infiltrative disease process (myocarditis/cardiomyopathy). The medium sized pericardial effusion was again shown, as was the normal origin of the coronary arteries and mild mitral valve regurgitation without any dilation of the left atrium. The 24-hour ECG recording did not reveal any noteworthy arrhythmias or conduction disturbances. Further evaluation of the ovaries by the gynaecologists refuted the possibility of ovarian malignancy.

The diagnosis of effusive constrictive pericarditis was made and the patient was treated with diuretics, $\beta$-blockers and corticosteroids (prednisone $1 \mathrm{mg} / \mathrm{kg} /$ day $\mathrm{IV})$. A low dose of angiotensin converting enzyme (ACE) inhibitor was also prescribed. Patient functional status improved significantly over the following days. The symptoms of dyspnoea on exertion and fatigue were abolished. Further, 16 days after admission, the patient was discharged with instructions for close follow-up at the Cardiology Department. At discharge blood tests showed microcytic anaemia of the same level as on admission, but inflammation markers and transaminase values were normal, as were BNP and proBNP. Heart ultrasound prior to discharge confirmed that LV systolic function was normal (estimated ejection fraction - EF $60 \%)$. Her next outpatient appointments with the Cardiology Clinic were arranged initially at monthly intervals. At follow-up her cardiac function remained normal with no fluid accumulation up to 1 year post discharge. She remains on $\beta$-blocker treatment (carvedilol $6.25 \mathrm{mg}$ bid), prednisolone at low doses ( $5 \mathrm{mg} /$ day), and hydroxychloroquine in accordance with the rheumatological consultations. There was no necessity of application of diuretics apart from a modest dose of spironolactone (12.5 mg od).

\section{Discussion}

Cardiovascular disease has recently been acknowledged as a primary cause of morbidity and mortality in SLE [2], and the heart is frequently involved in this systemic disease: very sensitive methods of cardiovas- 
cular investigation have found the prevalence of cardiac involvement to be $>50 \%$. In the past, cardiac manifestations were severe, often leading to death, and they were frequently found in post-mortem examinations. Nowadays, cardiac manifestations are often mild and asymptomatic, and they can be recognized by echocardiography and other non-invasive tests [3]. All three layers of the heart - pericardium, myocardium and endocardium - can be involved due to lupus cardiac involvement. Heart valve abnormalities (vegetations and/or thickening) are the most frequent cardiac manifestations of APS. These alterations, classically known as Libman-Sacks endocarditis, consist of verrucous endocarditis of valve leaflets, papillary muscles and the mural endocardium, originally described in SLE patients [4]. Heart valve lesions (vegetation, valve thickening and dysfunction) are frequently reported in patients with APS with or without SLE and in those with APS alone [5]. Finally, epidemiological studies show an increase of cardiac and cerebrovascular events in patients suffering from systemic autoimmune diseases, and autopsy studies further corroborate the notion that an accelerated atherosclerotic process is responsible for such manifestations $[6,7]$. These observations support a possible role of autoimmunity in the genesis of atherosclerosis that may have clinical or subclinical features. It is interesting that cardiovascular diseases (CAD) are described with a prevalence ranging from 6 to $10 \%$, and, in SLE patients, the risk of developing any CAD is 4-8 times higher than in controls [8, 9].

Pericarditis is the most studied cardiovascular manifestation of SLE, although often not evident clinically, and it is included in the American College of Rheumatology classification criteria for SLE. The pericardium can be involved by acute and chronic inflammatory changes. The reported prevalence of pericardial abnormalities, detected by echocardiographic studies, ranges from 11 to $54 \%$ [10]. Clinical (symptomatic) pericarditis is estimated to occur in $25 \%$ of SLE patients at some point in the course of their disease. Asymptomatic pericardial effusion is clearly more common than clinical pericarditis: in fact, $40 \%$ of unselected patients with SLE have pericardial effusion, detected using echocardiography. Pericardial involvement appears more frequently at SLE onset or during SLE relapses, although it can occur at any time of the disease. Pericarditis usually appears as an isolated attack or as recurrent episodes [3].

Signs and symptoms of acute pericarditis include a typical precordial or substernal chest pain, usually positional (aggravated by lying down), often with a pleuritic component, sometimes with dyspnoea; moreover, patients may have fever, tachycardia and decreased heart sounds; pericardial rubs can be heard but usually are rare, perhaps because they are present often for only a few hours and are missed. The diagnosis can be confirmed by ECG findings. Patients with pericardial effusion (as opposed to thickening) are more likely to have pericardial pain and active lupus elsewhere; when present, pericardial effusions are usually small and do not cause haemodynamic problems [11, 12]. As echocardiography is the standard method to identify pericardial abnormalities, it is advisable to perform it in SLE patients at regular intervals. Complications of pericarditis, such as cardiac tamponade, constrictive pericarditis and purulent pericarditis, are rare, and invasive procedures (pericardiocentesis or pericardial window) are scarcely needed.

Non-steroidal anti-inflammatory (NSAID) drugs and/ or corticosteroids are the first line of treatment in mild pericarditis. Colchicine alone or as adjunctive therapy to NSAIDs can be of further value. Finally, intravenous corticosteroids may be necessary in severe cases or when tamponade is present, while in patients with recurring pericarditis, chronic immunosuppression may be effective. Specific medical treatment should be targeted at the cause of the disease as much as possible. However, the cause of pericardial diseases may be varied, and in clinical practice the etiological search is often inconclusive and most cases are classified as idiopathic.

\section{Summary}

In the case presented a middle aged female with known medical history of SLE was referred because of pericardial effusion associated with mild symptoms of dyspnoea on exertion and fatigue. Final diagnosis was effusive constrictive pericarditis secondary to SLE. Medical treatment sufficed to improve patient clinical status, and in long-term follow-up the patient is asymptomatic with no signs of disease recurrence. Heart involvement in SLE is not unusual, and the attending physician should suspect and investigate for cardiac involvement as symptoms may be relatively mild, even in advanced disease, and early treatment may drastically alter the disease course and prognosis of those affected.

The authors declare no conflict of interest.

\section{References}

1. Turiel M, Peretti R, Sarzi-Puttini P, et al. Cardiac imaging techniques in systemic autoimmune diseases. Lupus 2005; 14: 727-731.

2. Tincani A, Rebaioli CB, Taglietti M, Shoenfeld Y. Heart involvement in systemic lupus erythematosus, anti-phospholipid syndrome and neonatal lupus. Rheumatology (Oxford) 2006; 45 (Suppl 4): iv8-iv13.

3. Doria A, laccarino L, Sarzi-Puttini P, et al. Cardiovascular involvement in systemic lupus erythematosus. Lupus 2005; 14 : 683-686. 
4. Cervera R, Piette JC, Font J, et al. Antiphospholipid syndrome: clinical and immunologic manifestations and patterns of disease expression in a cohort of 1,000 patients. Arthritis Rheum 2002; 46: 1019-1027.

5. Moyssakis I, Tektonidou MG, Vasilliou VA, et al. Libman Sacks Endocarditis in Systemic Lupus Erythematosus: prevalence, associations and evolution. Am J Med 2007; 120: 636-642.

6. Salmon JE, Roman MJ. Accelerated atherosclerosis in systemic lupus erythematosus: implications for patient management. Curr Opin Rheumatol 2001; 13: 341-344.

7. Van Doornum S, McColl G, Wicks IP. Accelerated atherosclerosis. An extraarticular feature of rheumatoid arthritis? Arthritis Rheum 2002; 46: 862-873.

8. Rhew EY, Ramsey-Goldman R. Premature atherosclerotic disease in systemic lupus erythematosus - role of inflammatory mechanisms. Autoimmun Rev 2006; 5: 101-105.

9. Matsuura E, Lopez LR. Autoimmune mediated atherothrombosis. Lupus 2008; 17: 878-887.

10. Doria A, Petri M. Cardiac involvement in systemic lupus erythematosus. In: Doria A, Pauletto P (eds.). The heart in systemic autoimmune disease. Elsevier, Amsterdam 2004; 146-162.

11. Leung WH, Wong KL, Lau CP, et al. Cardiac abnormalities in systemic lupus erythematosus: a prospective M-mode, cross-sectional and Doppler echocardiographic study. Int J Cardiol 1990; 27: 367-375.

12. Kalke S, Balakrishanan C, Mangat G, et al. Echocardiography in systemic lupus erythematosus. Lupus 1998; 7: 540-544. 